\title{
Advanced In-cycle and Cycle-to cycle On-line adaptive Control for Thermoforming of large Thermoplastic Sheets
}

\author{
Patrick Girard, Robert di Raddo \\ Industrial Materials Institute , National Research Council of Canada, 75 de Mortagne, Boucherville, Qc, Canada, J4B 6Y4 \\ Vince Thomson, Benoit Boulet \\ McGill University, Mechanical Engineering, 817 Sherbrooke St. W, Montreal, Qc, Canada, H3A 2K6 \\ McGill University, Electrical and Computer Engineering, 3480 University St., Montreal, Qc, Canada, H3A 2A7
}

Copyright @ 2004 SAE International

\begin{abstract}
This paper presents a control system for the thermoforming of large sheets. The system has three main purposes: Its primary purpose is for cycle-to-cycle control of the sheet temperature map as predicted by the thermoforming simulation. Its secondary purpose is to tune the simulation to the actual process through online characterization of some of the material and machine characteristics so that the simulation's predictions are reconciled with the machine sensors outputs. Lastly it can characterize on-line a number of independent and fast acting sub-models that allow for in-cycle control of some process parameters.
\end{abstract}

\section{INTRODUCTION}

Large sheet thermoforming (Figure 1) is widely used for the manufacture of large parts such as twin sheet formed gas tanks, body panels and windshields. In its present state simulation software can predict with good accuracy the temperature map and internal temperature profile that is required by the small processing window of modern specialty materials. Henceforth a primary requirement for consistent part quality is that the actual sheet temperature distribution before forming meets precisely these predictions.

However, there are a number of problems to be addressed before a simulation can be efficiently integrated into process control:

- Material properties used in the simulation are not measured at actual operating conditions and can vary from batch to batch. For example sheet sag in oven varies with the level of material frozen-in stresses brought on by sheet calendering.

- The power radiated from heating elements to sheet zones varies with element temperature and element output varies with life.

- Computing time for a simulation is much greater than cycle time, precluding its application to incycle control, which is required for adequate quality, especially during the start-up phase.

The model based control system proposed here (Figure 2) uses three levels of control to meet these requirements.

- The foundation of the system is a simulation software that operates at the cycle-to-cycle time frame. This allows for an initial guess that is sufficiently close to the actual operating conditions that linear adjustments can be used to implement the control model. The simulation model must be sufficiently sophisticated that it allows for reverse engineering of the required process parameters. We are presently using for this purpose the BlowSim Thermoforming simulation software developed at the Industrial Materials Institute (IMI).

- Since sensor data will generally be treated on a distributed system, numerous measurement and transmission errors are to be expected and an error detection and recovery scheme is required at this level. A diagnostic system is being implemented at this point. 
- The most innovative part of this system is the in-cycle control and characterization system that constantly monitors drifts and variations in the system parameters. It consists of a number of fast acting distributed system that can characterize the following properties:

- Level of initial frozen-in stresses in sheet.

- Reflection coefficient during heating in the oven.

○ Material Heat capacity.

- Material radiation absorption.

- Element to zone view factors.

$\circ \quad$ Elements heating transfer functions.

A multi-agent system that can deal with multiple data streams and multiple process sub-models supervises these models and updates the simulation model as required.

A team consisting of $4 \mathrm{IMI}$ personnel, 2 McGill University professors, 2 McGill post-docs and 3 graduate students is working on the implementation of this control system. The system was initially developed at IMI's large scale laboratory on a Monarch twin sheet thermoforming machine (Figure 3) that has dual 6' square ovens and 504 heating elements in reconfigurable zones. The system is currently being installed at PlastikMP, a Quebec, Canada Thermoformer (see additional sources).

\section{MODEL BASED CONTROL AND SIMULATION TUNING}

The control system tries to find the heating zones temperature map that will reconcile the temperature map as predicted by the simulation with the $2 D$ temperature map as measured on the sheet by a Raytek Thermalert MP50 infrared scanning thermometer at the exit of the oven (figure 4). An interface to FormSim was developed that allows it to read directly the output of the Raytek scanner and generate the initial mesh for the simulation (figure 5). This common mesh makes it much easier to compare subsequent $2 \mathrm{D}$ temperature scans for system adjustment.

\section{SIMULATION TUNING AND CYCLE-TO-CYCLE CONTROL}

An initial simulation is started from a temperature zone map (figure 6) that reproduces the actual zone map of the machine. The control system actually controls the amount of power which is sent to each element. This power however needs to be displayed to the operator as element temperatures. This is made possible by identifying the steady state power transfer function for a typical element (figure 7) which relates input wattage and various element temperatures in steady state.

In order to tune the system we need to find the relation $W_{i j}$ between the power consumed by heating element $i$ and the radiative power that it sends to sheet zone $j$ (figure 8). This is explicited by:

$$
[\text { Welement }] \quad\left\lfloor W_{i j}\right\rfloor=\left\lfloor W_{\text {zone }}\right\rfloor
$$

A list of all symbols used is given at the end of this document.

All of the heating zones (48) on one side of the Monark machine have been implemented with Amploc 25A current sensors in order to monitor the instantaneous power consumption of each zone. The initial values for $\mathrm{W}_{\mathrm{ij}}$ can be obtained from the simulation. Subsequently the element temperature values that match the process are found by adjusting the $\mathrm{W}_{\mathrm{ij}}$ matrix using ThermoDesign, a constrained optimization software developed at IMI.

While this takes place the properties characterized on line at the actual operating conditions are used to adjust the input of the simulation, and the simulation is then tuned to match the actual sensor measured values.

Presently, because of controller warranty considerations the thermoforming machine is operated in open loop, which means that the proposed changes in element temperatures are presented to the operator who then enters the new set points manually. A later version could implement closed loop (i.e. direct transfer to the machine) once the diagnostics and error correction system has been set-up.

\section{DIAGNOSTICS AND ERROR CORRECTION SYSTEM}

The diagnostics and error correction system are presently being developed. The error correction system is required because of the distributed nature of the system which encourages noise interference. The diagnostic system will be presented separately.

\section{CHARACTERIZATION AND IN-CYCLE CONTROL}

Because of variations in material properties from batch to batch it is imperative that a control system incorporates methods to follow any such variations, so that they can be used to adjust the simulation's input. The list of parameters that can be identified is given above. Three examples will be presented here. 
Prediction of the temperature in the sheet at any time and depth

The in-oven temperature sensor information can be used to generate sub-models that can for example predict [2] the temperature at any depth and time in the material from the in-cycle history of heating using:

$$
\boldsymbol{\theta}_{t, d}=\exp \left(\frac{a_{1}}{\left(a_{3}{ }^{+} d\right)_{t}}+a_{2}\right)
$$

Where $\theta$ is the temperature, $t$ and $d$ are respectively the time and depth and the $a_{i}$ are fitted coefficients.

\section{Characterization of the rheological behaviour of the} material

Modelling the rheological behaviour of the material correctly is a major challenge for the simulation since it is very difficult to obtain reliable data for the conditions at which thermoforming is performed, especially with regard to sheet sag prediction [3]. A technique that uses an existing mould to blow a bubble is presented in figure 9. In this case a simple circular mould was used to minimize the influence of the geometry (figure 10). The bubble is then formed by pressure application above the mould (figure 11). The shape of the bubble is measured by two laser ranging sensors And two pressure sensors follow the corresponding development of the bubble pressure. Figure 12 presents the test results with the zone in which the stress to shear rate relationship of the material can be evaluated. A generalized Maxwell equation was found to be the best suited to fit the operating conditions.

$\tau_{i j}(t)=\int_{-\infty}^{t} \sum_{j=1}^{N} G_{i}\left(\exp \left[-\left(t-t^{\prime}\right) / \lambda_{i}\right]\right) \dot{\gamma}_{i j}\left(t^{\prime}\right) d t^{\prime}$

Where $\mathrm{N}$ is the number of relaxation times.

Characterization of the internal Beer Lambert Radiation Absorption Coefficient

One of the most important variables for the thermoforming process is the radiation absorption of the material. A novel technique that can be implemented on-line if two thermocouples can be inserted in a nonsensitive area of the material is presented here.

The approach is based on the fact that on entry in the oven the sheet is at a uniform temperature and heating by conduction at a given depth takes some time governed by the diffusivity of the material. Hence an immediate increase in temperature can result only from radiative heating. Figure 13 presents the start of heating for a $12.5 \mathrm{~mm}$ thick HDPE sheet.

The 1D heating equation for the sheet is:

$$
\rho C_{p} \frac{\partial \theta}{\partial t}=k \frac{\partial^{2} \theta}{\partial x^{2}}+\frac{\partial\left(\dot{q}_{a b s}\right)}{\partial x}
$$

As the start of heating since the sheet is isothermal we can neglect the temperature gradient:

$$
\frac{\partial^{2} \theta}{\partial x^{2}}=0
$$

If $K$ is the absorption in a $1 \mathrm{~mm}$ thick layer the temperature increase rate at a depth of $3 \mathrm{~mm}$ is:

$$
\rho C_{p} \frac{\partial \theta}{\partial t}=F_{i} * \kappa(1-\kappa)^{2}
$$

At a depth of $5 \mathrm{~mm}$ :

$$
\rho C_{p} \frac{\partial \theta}{\partial t}=F_{i} * \kappa(1-\kappa)^{4}
$$

Which gives us $K$ with a very good precision since the temperature gradient is integrated by curve fitting over a period of time:

$$
\frac{\frac{\partial \theta}{\partial t}(3 \mathrm{~mm})}{\frac{\partial \theta}{\partial t}(5 \mathrm{~mm})}=(1-\kappa)^{-1}
$$

Comparison of initial and optimized temperature map and bubble sag

Figure 14 presents the initial simulated temperature profile using the mesh generated from the $2 \mathrm{D}$ temperature scanner output. The temperature is then remapped on the sheet. Since the system controls the sheet temperature using in-oven IR thermometers and not the element temperature as is commonly used, the temperature control is much more precise as explicited in [4].

One of the predicted parameters of the simulation is the in-oven sheet sag, which can be used as a control parameter [3]. Figure 15 presents the preliminary results for the predicted and measured bubble sag. The deviation at longer times can be related to a problem with the simulation model. It must be noted that sag prediction is currently very unreliable.

\section{CONCLUSIONS}

- Cycle-to-cycle system control tries to realize at the oven's exit the temperature map as predicted by the tuned simulation.

- In-cycle control is implemented using distributed sub-models that characterize on-line process drifts and variations. The data thus identified is 
then used to tune the simulation. This allows the system to follow ambient and batch to batch variations.

- The tuning process provides valuable insight about the validity and accuracy of the internal models used by the simulation.

\section{ACKNOWLEDGMENTS}

We would like to thank Philippe Courtois of IMI for realizing the experiments and simulations in this article, and Guy Gauthier of McGill University for figure 7.

\section{REFERENCES}

1. "On-line Hybrid Model-Based Tuning of Simulation Provides Soft Sensors for the Estimation of Sheet Temperature Distributions in Thermoforming"; P. Girard, V. Thomson, B. Hou, A. Yousefi and R. DiRaddo; ANTEC 03; Nashville, TN

2. "Simulation and Experimental Hybrid Model-based Soft Sensors for Intelligent Control of Forming Operations", Girard, P., Hou, B., Thomson, V., Yousefi A. and DiRaddo, R.W.; The Fourth International Conference on Intelligent Processing and Manufacturing of Materials, Sendai, Japan, May 2003.

3. "Improving the Prediction of Thermoforming Sag in Simulations through experimental Validation of the Transition Model"; Linda Pecora; Patricia Debergue; Patrick Girard; Christian de Grandpré, ANTEC 04, Chicago, MI.

4. "A NEW ABSORPTION BASED MODEL FOR SHEET REHEAT IN THERMOFORMING"; Guy Gauthier; Mark Ajersch; Ammar Haurani; Benoit Boulet; Robert DiRaddo; To be published at ANTEC 05.

\section{CONTACT}

Patrick Girard, Patrick.girard@nrc.ca
Michel Pelchat, PlastikMP, 250 Gouin, C.P. 1218, Richmond, Quebec, Canada, J0B2H0

\section{DEFINITIONS, ACRONYMS, ABBREVIATIONS}

$\boldsymbol{\theta}$ temperature

$t \quad$ time

d depth

$\rho \quad$ Density

Cp Heat capacity

$q_{a b s} \quad$ Absorbed heat

k Heat conductivity

$\mathrm{Fi} \quad$ Incident heat flux on the sheet

$\kappa \quad$ Internal coefficient of radiation absorption

$\tau_{\mathrm{ij}} \quad$ Shear stress

$\mathrm{G}_{\mathrm{i}} \quad$ Relaxation modulus

$\gamma \quad$ Shear rate

$\lambda \quad$ Relaxation time

W Power

\section{ADDITIONAL SOURCES}




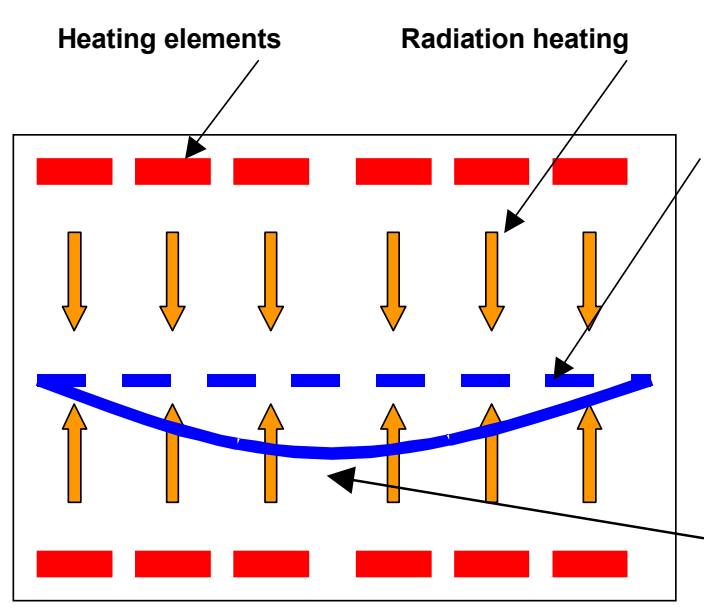

Oven

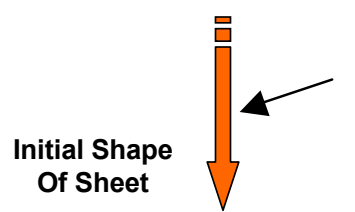

In oven sag and temperature measurement
Transfer to Forming Station

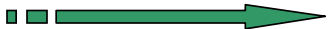

2D Temperature scan

at exit of oven

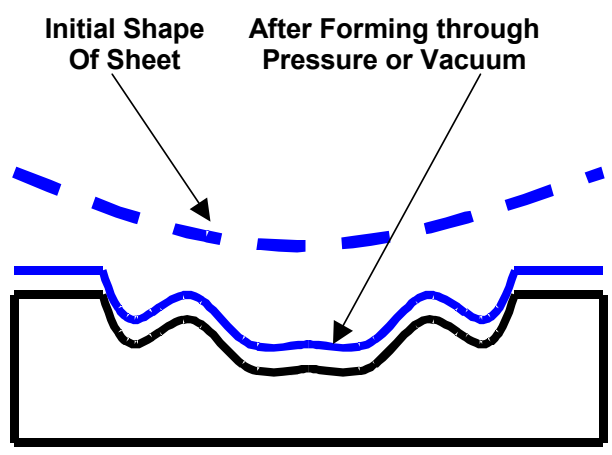

Forming Station

Figure 1: The thermoforming process

Control Loop

between models and

process

Diagnostic

and error correction level

Process Models

for in-cycle and cycle-to-

cycle control
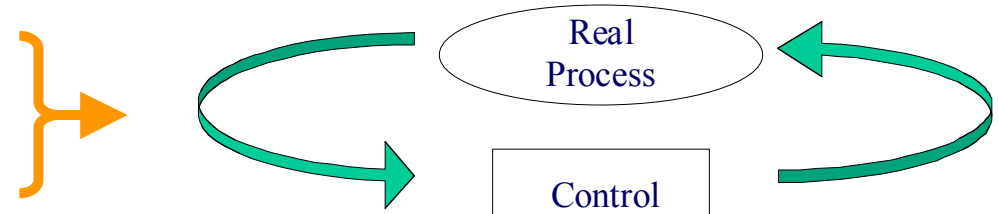

OUT
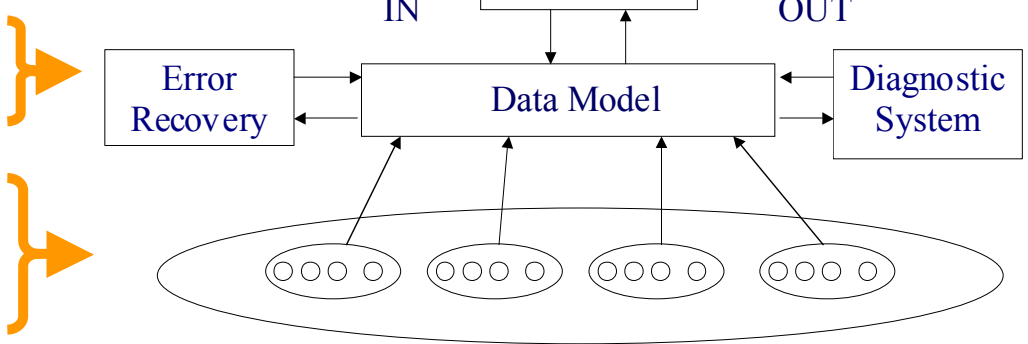

Process model and submodels

Figure 2: Three Level in-cycle and cycle-to-cycle Control with Diagnostic System

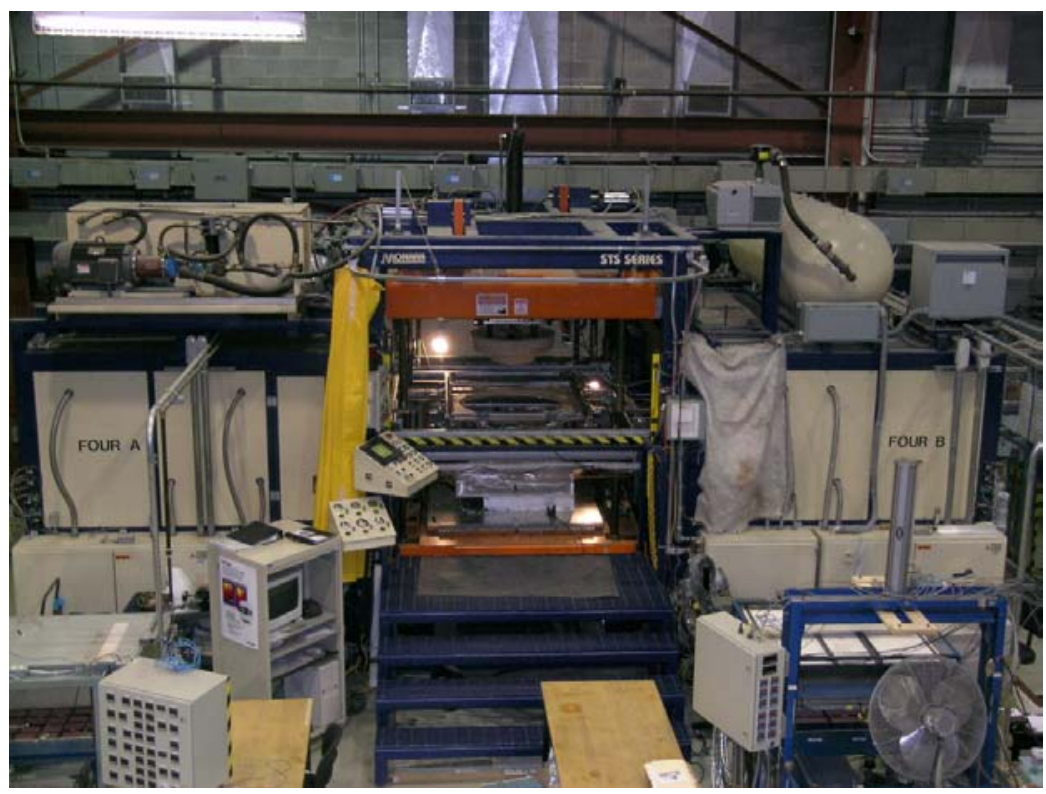

Figure 3: Monark Twin Sheet Thermoforming Machine 


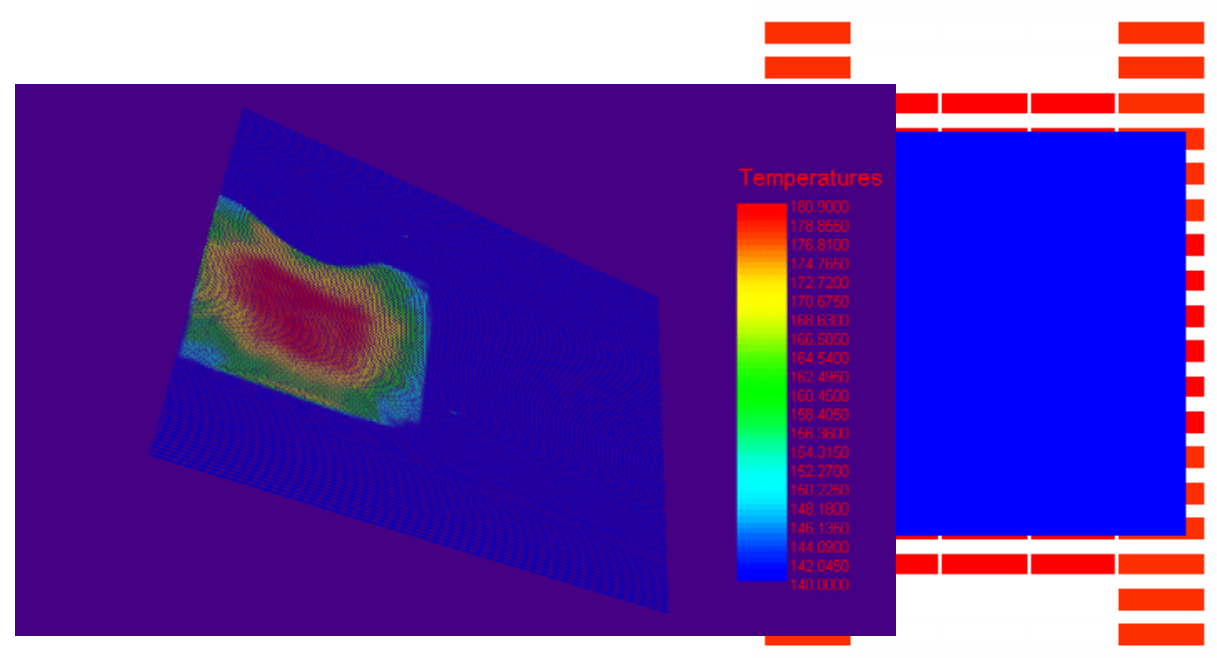

Temperature_(oC)

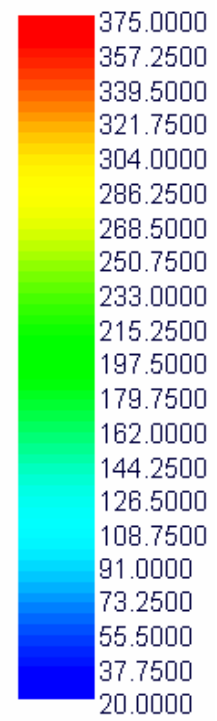

Figure 4: 2D Scan of Sheet Temperature at Exit of Oven as read by the simulation: Note that the zone in the upper area is out of temperature.

\section{Oven B: Top Zone Layout}

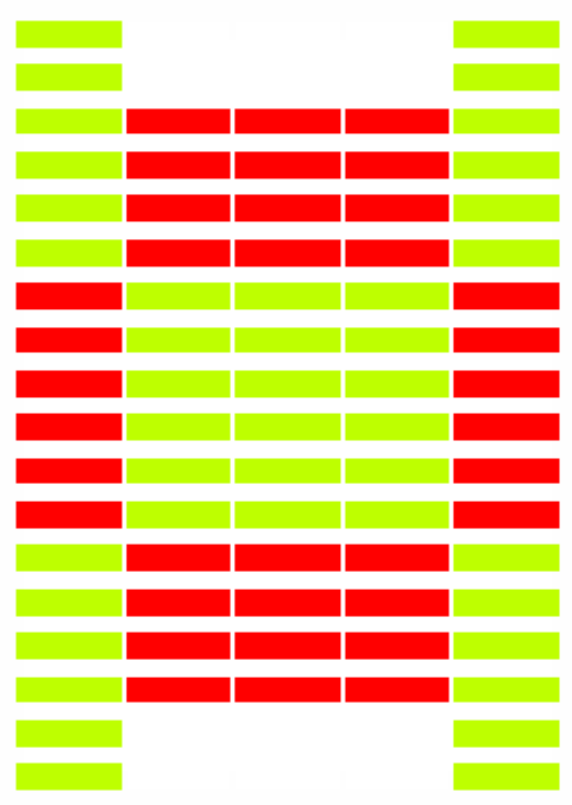

Figure 5: Generation of Sheet for Simulation from 2D scan

\section{Oven B: Bottom Zone Layout}
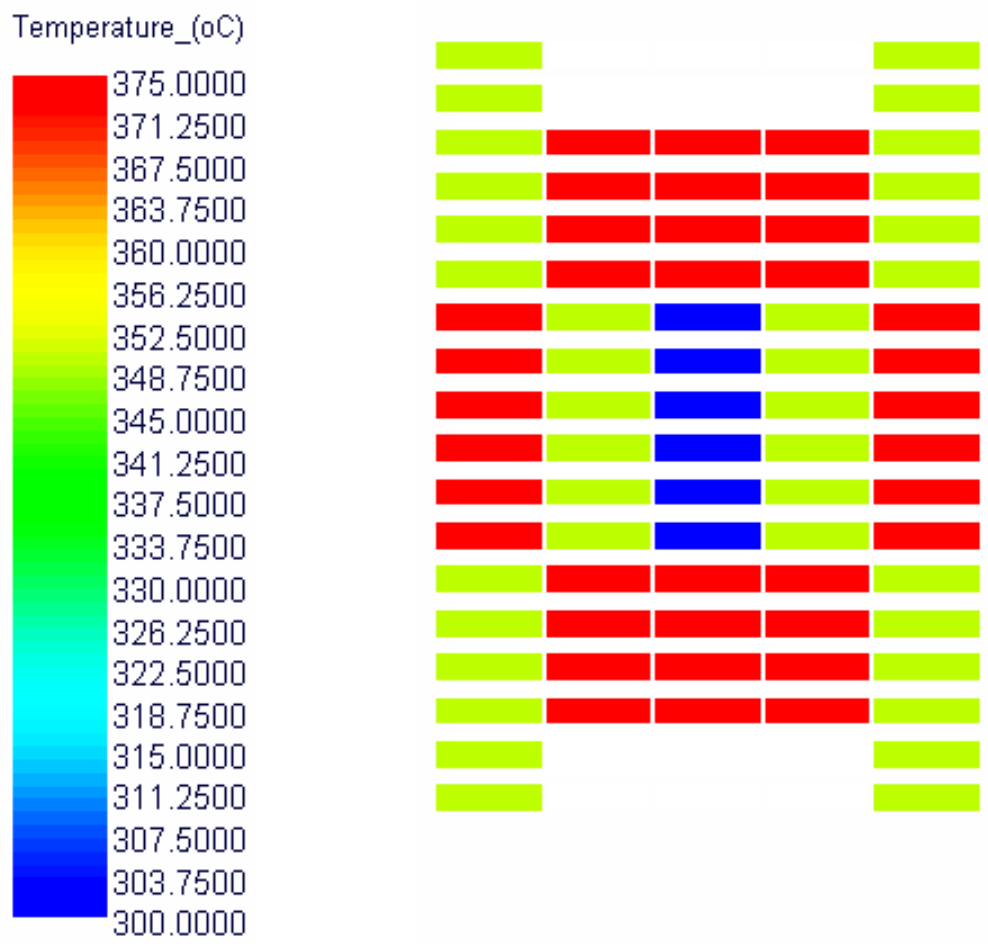

Figure 6: Initial Heating Element Layout for Simulation

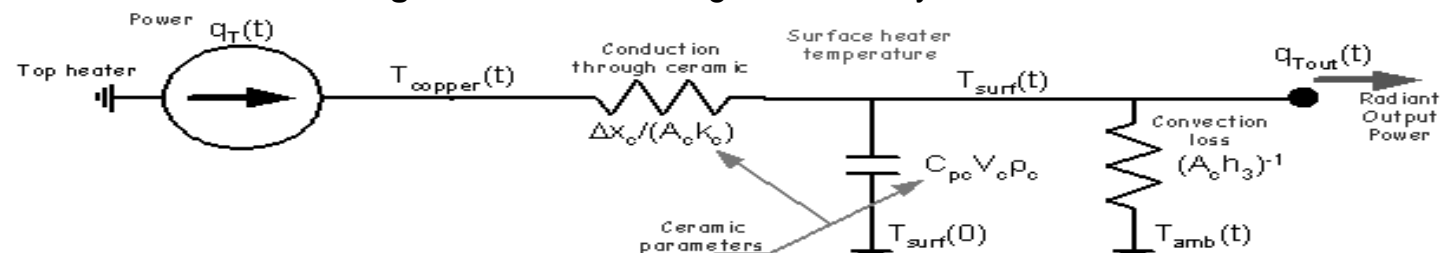


Figure 7: Heating Element Transfer Function relates Temperatures and Power Input

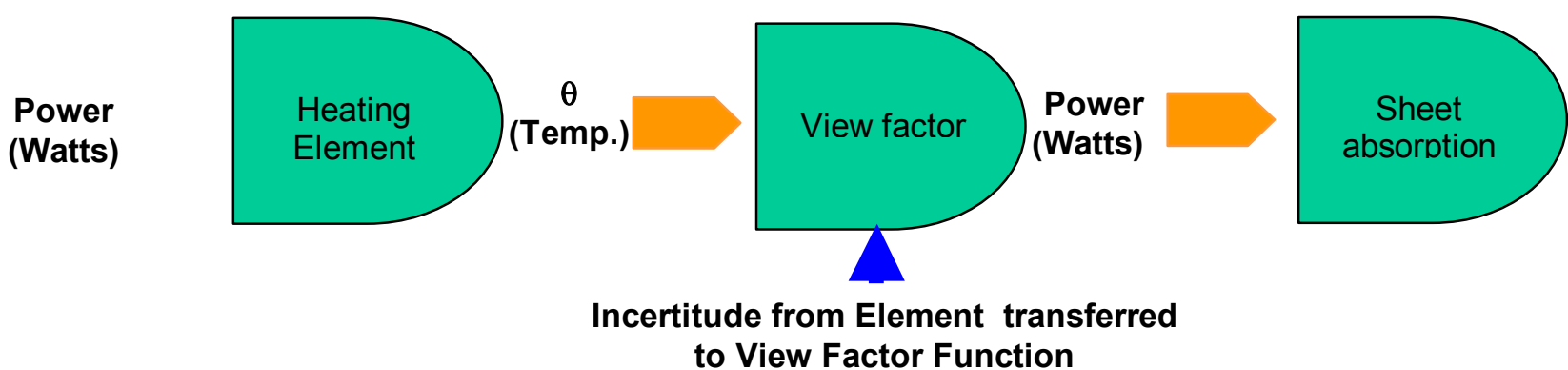

Figure 8: Power Transfer in Oven from Heating Element to Sheet

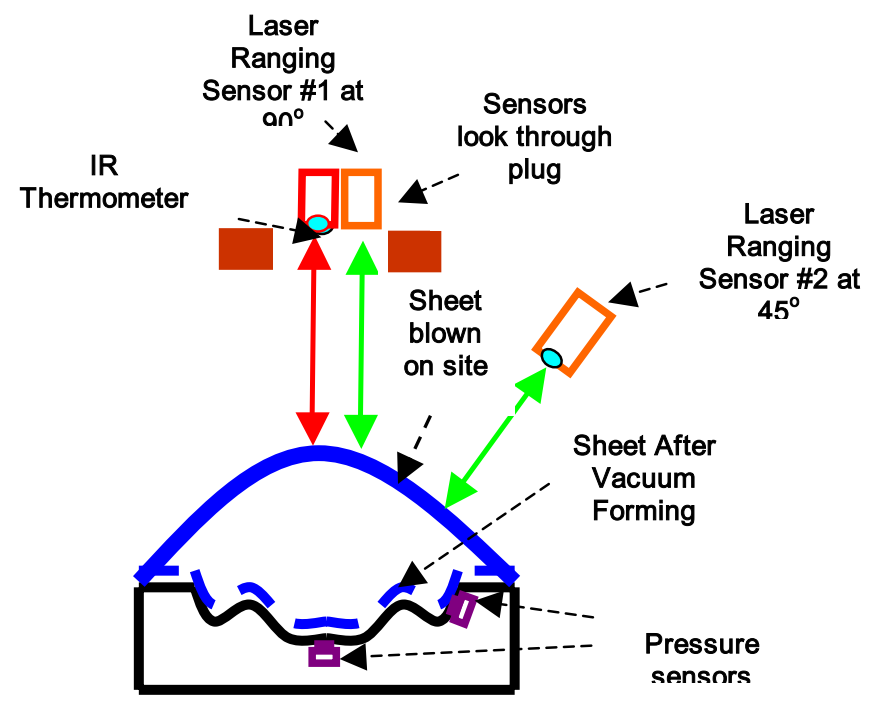

Figure 9: Forming Station instrumented for Material Characterization

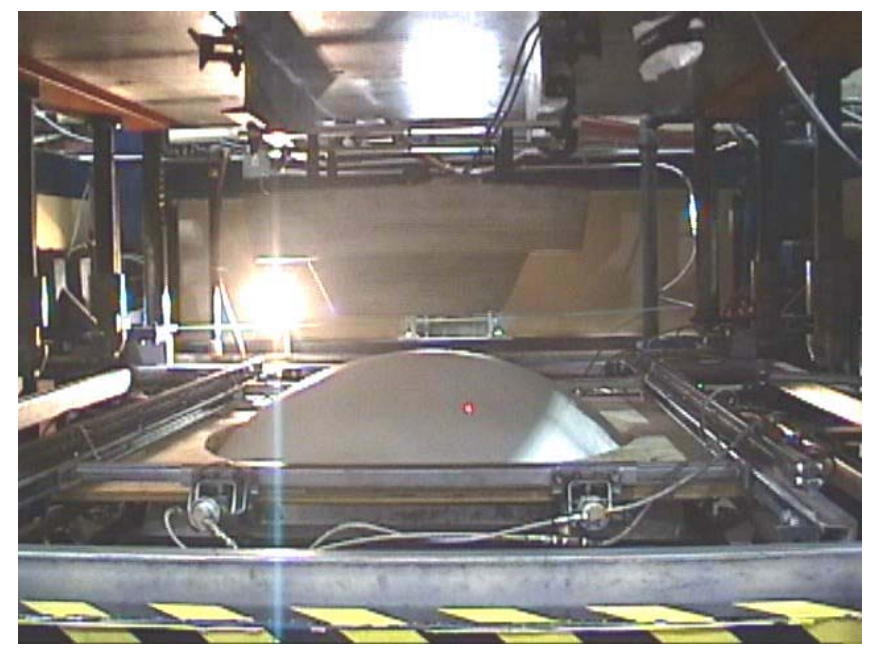

Figure 11: Bubble being blown above Mould with Laser ranging Spot

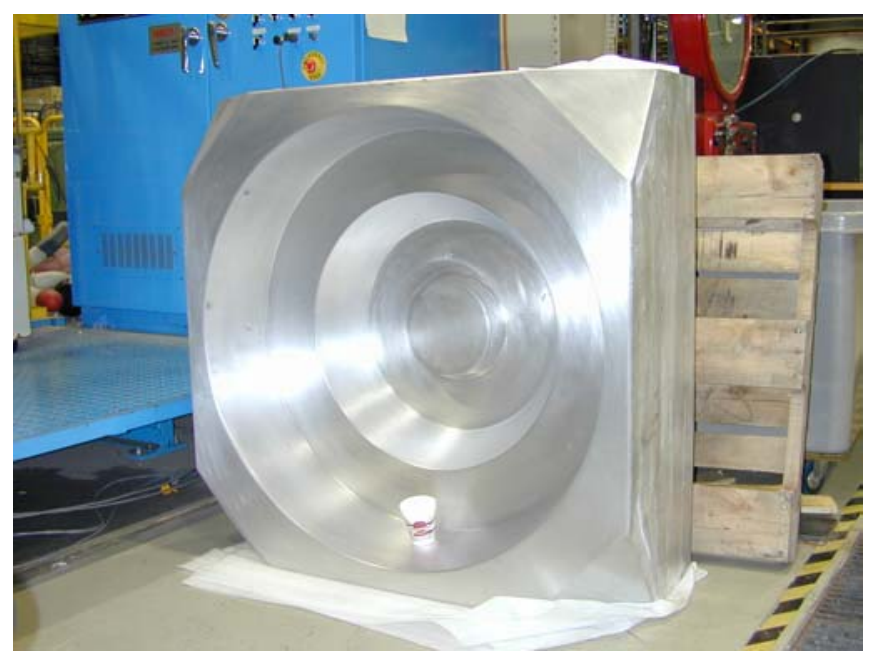

Figure 10: Thermoforming Mould

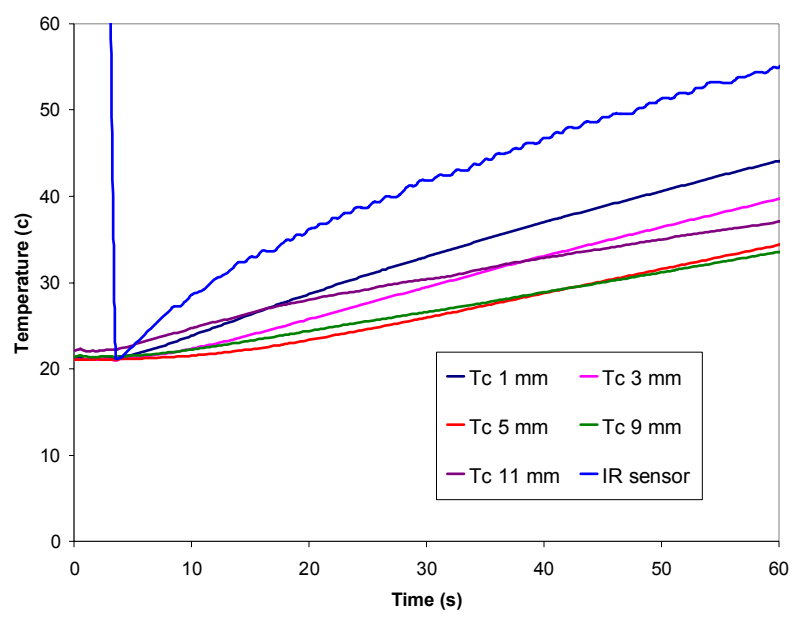

Figure 13: Identification of Radiation Absorption at Start of Heating for a $12.5 \mathrm{~mm}$ Thick HDPE Sheet 
$\mathbf{m m}$

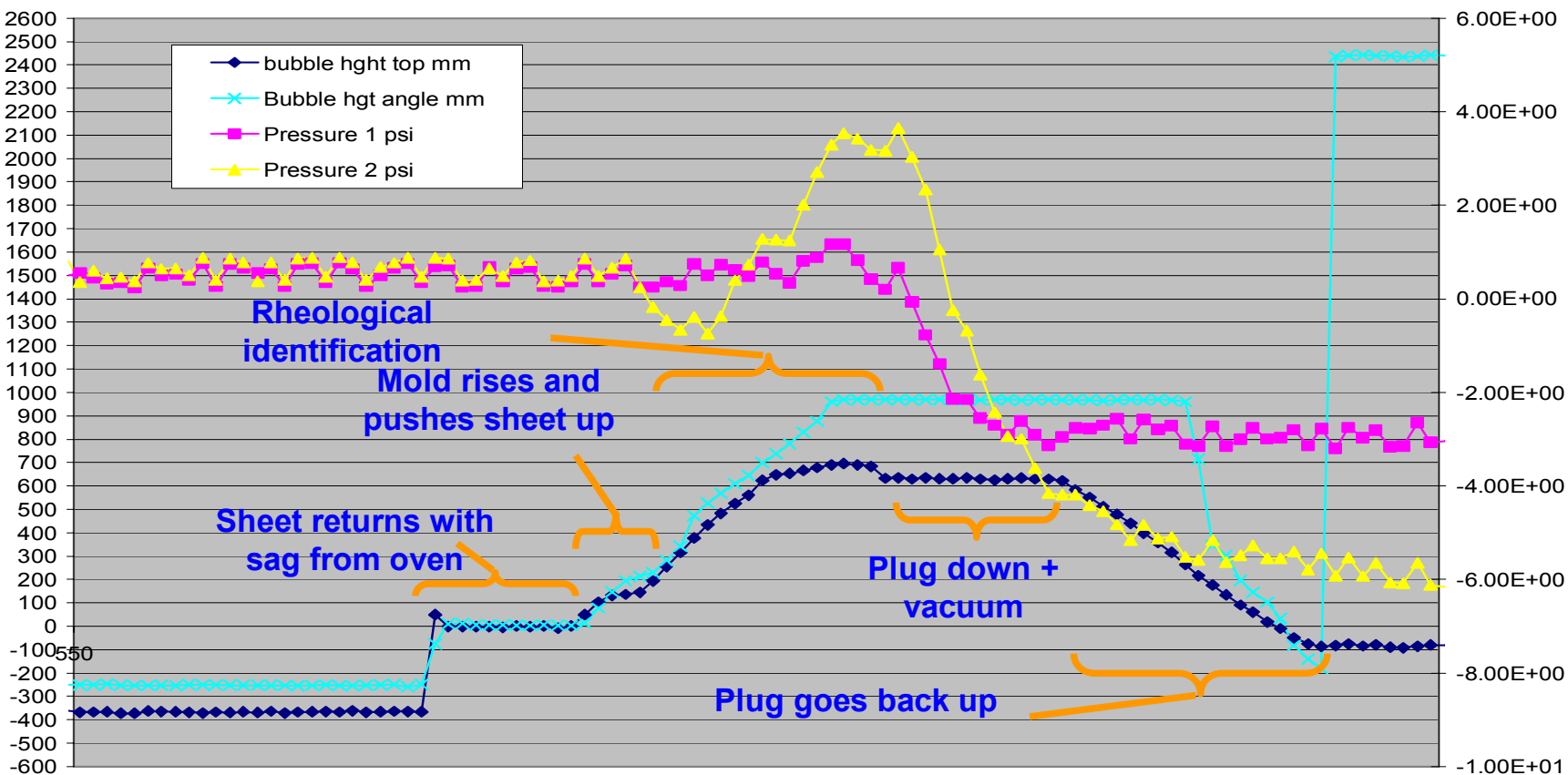

Figure 12: Rheological Identification from Bubble Forming

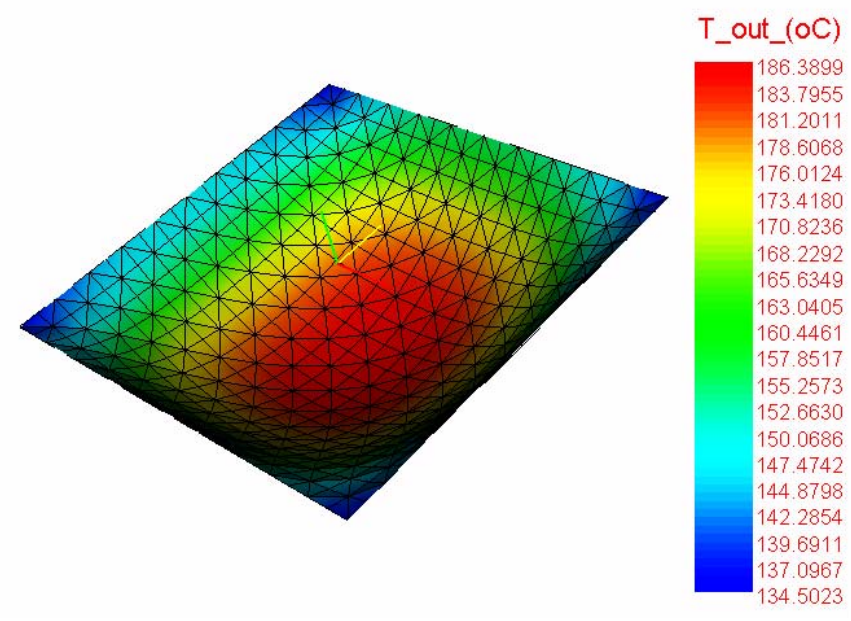

Figure 14: Initial sheet temperature as predicted by the simulation with layout of fig. 6 .
Sag of 1/4" (6.25mm) HDPE Sheet

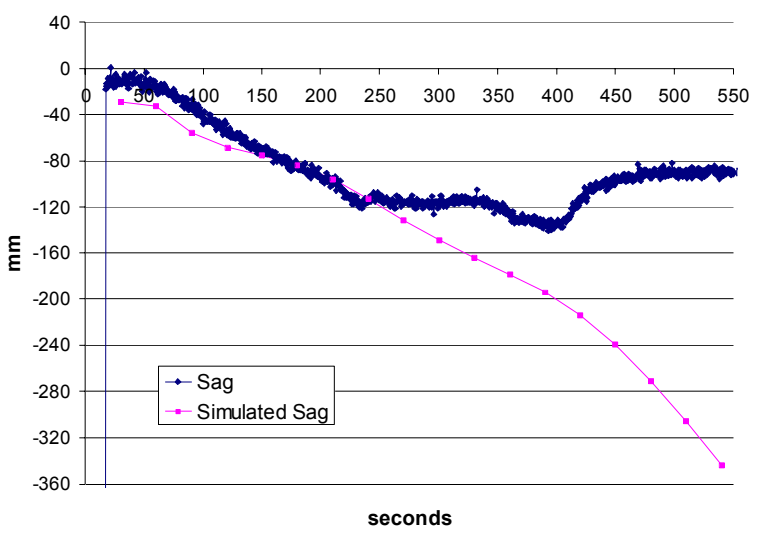

Figure 15: Initial experimental and predicted in-oven bubble sag 